\title{
Oral Health Care and Treatment Needs in Postmenopausal Women
}

\author{
Chaitali Hambire ${ }^{1}$, Umesh Hambire ${ }^{2}$
}

\begin{abstract}
The relationship between oral and general health is bidirectional. During menopause, there is permanent cessation of menstrual cycle with a decrease in female hormonal level. This results in changes in general health, like dryness of the vagina, hot flashes, and dermatological, psychological, and cognitive changes. The oral manifestations include xerostomia, burning mouth syndrome, and periodontal and gingival diseases. The histological similarity between the oral and the vaginal mucosa can explain the similarity in their symptoms.

Aims and objectives: To review the literature regarding oral health care and treatment needs of women after menopause and to guide gynecologists, dentists, and general practitioners in the principles of treatment of women with menopause-related oral health issues.

Methods: The PubMed, EBSCO, and Cochrane database were searched until June 2020 with keywords like "menopause,"'menopausal problems," "postmenopausal health issues," "oral health," "periodontal problems," "dental health," "dental disorders," "saliva," "burning mouth syndrome," "glossodynia," "xerostomia,"and "menopause hormone therapy (MHT)."

Results: Estrogen receptors are present in the periodontium and salivary glands. This may account for increased incidences of periodontal diseases, xerostomia, and burning mouth syndrome in perimenopausal and postmenopausal women, as there is a sharp decline in female steroid hormones. Xerostomia and glossodynia were the most common oral symptoms in women during and after menopause. MHT has not shown much effect in reducing oral symptoms.

Conclusion: Dentists and gynecologists should work together to help perimenopausal and postmenopausal women in the reduction of their oral health problems and encourage them to maintain good oral hygiene and diet practices. There is a need for randomized controlled trials related to the role of hormone replacement therapy on oral health.

Keywords: Menopause, Menopause hormone therapy (MHT), Oral health, Periodontitis, Xerostomia.

Journal of Oral Health and Community Dentistry (2021): 10.5005/jp-journals-10062-0087
\end{abstract}

\section{INTRODUCTION}

Menopause is defined as a physiologic process occurring in women in the fifth decade of life involving permanent cessation of menstruation. Menopause is a gradual process occurring over an extended period of time with interruptions of 1 to 3 months. According to World Health Organization, 12 consecutive months of amenorrhea, for which there is no other obvious pathological or physiological cause, is considered to be natural menopause. Recent studies have reported that most women take 2 to 6 years to reach menopause while some can take about 8 years. ${ }^{1}$

\section{Menopause Pathophysiology}

During a normal menstrual cycle, the theca and the granulosa cells of ovarian follicles are stimulated by the luteinizing hormone (LH) and follicle-stimulating hormone (FSH), secreted from the anterior pituitary. The estrogen produced by the granulosa cells exerts a negative feedback to the brain and inhibits the production of FSH. Of multiple follicles, only a single-dominant follicle continues to grow and produces an increasing amount of estrogen. After a period of estrogen exposure, positive feedback (superimposed on negative feedback) of estrogen stimulates a surge of LH and FSH. The surge of $\mathrm{LH}$ triggers the ovulation from the dominant follicle. ${ }^{2}$

Recent studies support the hypothesis that there is relative hypothalamic-pituitary insensitivity to estrogen in aging women. In postmenopausal women, a sufficient level of estrogen is not able to inhibit LH to provide a negative feedback to the central nervous system, maybe the "opening of the negative feedback loop

\footnotetext{
1,2Department of Pediatric Dentistry, Government Dental College and Hospital, Aurangabad, Maharashtra, India

${ }^{2}$ Department of Mechanical Engineering, Government College Of Engineering, Aurangabad, Maharashtra, India

Corresponding Author: Chaitali Hambire, Department of Pediatric Dentistry, Government Dental College and Hospital, Aurangabad, Maharashtra, India, Phone: +91 9422284546, e-mail: chaitalikmirajkar@ gmail.com

How to cite this article: Hambire C, Hambire U. Oral Health Care and Treatment Needs in Postmenopausal Women. J Oral Health Comm Dent 2021;15(1):24-29.

Source of support: Nil

Conflict of interest: None
}

between ovarian estrogen and pituitary LH." There is a decrease in the level of progesterone along with an increase in the level of testosterone,particularly androstenedione. These androgens compensate for the deficiency of estrogen by the production of estrone from peripheral fat or adipose tissue. As a result, there is an increase in total fat, visceral and subcutaneous abdominal fat. There is a reduction in total and peripheral lean body mass. This causes a decrease in insulin synthesis and an increase in insulin resistance, which results in altered glucose metabolism. As a result,postmenopausal women suffer from an increased risk of diabetes and other metabolic disorders. Altered lipid metabolism, such as increased lipid synthesis and decreased lipolysis, results in an increased risk of cardiovascular 
diseases. Headaches, hypertension,tachycardia, and arrhythmias are some cardiovascular problems. ${ }^{3}$

During bone remodeling, the osteoclasts resorb the mineral content of the bone, after which they undergo apoptosis. The osteoblasts deposit collagen which undergo mineralization to form a new bone. This process is controlled byPTH (parathyroid hormone), calcitonin, 1,25(OH)2-vitamin D3, and estrogen. Estrogen affects the bone through the following mechanisms: (1) decreasing the sensitivity of bone mass to PTH, thereby reducing bone resorption, (2) increasing the production of calcitonin, thus inhibiting bone resorption, (3) accelerating calcium resorption by the intestine, (4) reducing calcium excretion from the kidneys, and (5) estrogen can also have direct effects onthe bone through estrogen receptors. The important action of estrogen is to increase osteoprotegerin (OPG) secretion and decrease macrophage colony-stimulating factor (M-CSF) and RANKL (receptor activator of nuclear factor B ligand). Estrogen reduces osteoclastogenesis by suppressinginterleukin-1 (IL-1) and tumor necrosis factorand increasing the sensitivity of stromal cells/preosteoblasts to IL-1, thus suppressing M-CSF, RANKL, and perhaps most notably, IL-6. In addition, estrogen stimulates the production of OPG, the potent inhibitor of osteoclastogenesis. Estrogen also promotes osteoclastic apoptosis with the help of transforming growth factor $\beta$ (TGF $\beta$ ). During menopause, the decreased level of estrogen results in acceleration of osteoclastic activity without a corresponding increase in osteoblastic activity. This results in a net loss of bone. ${ }^{4}$

Decreased level of estrogen is also responsible for psychosomatic disorders, like hot flashes, neuralgias, headaches,paresthesias, mood swings, dysphoria, pruritus, vertigo,sleep disorders, and depression. It is also responsible for urological disorders, likediminished bladder capacity, micturition urgency, dysuria, pollakiuria, and suprapubic pain. ${ }^{5}$

\section{Effect of Female Sex Hormone on Oral TISSUES}

Various cytological studies have shown resemblance between oral mucosa, gingiva, and vaginal mucosa. Receptors for female sex hormones have been found in oral mucosa, periodontium, and salivary glands. Hence,estrogen deficiency during menopause is considered to be responsible for various oral health problems. These hormones affect the proliferation, differentiation, and growth of keratinocytes and fibroblasts in the gingiva. Their action can be explained by two theories. According to the first theory, these hormones alter the effectiveness of the epithelial barrier to a bacterial insult. Second theory suggests their effect on collagen maintenance and repair. ${ }^{6}$

Estradiol can induce cellular proliferation while depressing protein production in cultures of human premenopausal gingival fibroblasts. This cellular proliferation appears to be the result of a specific population of cells within the parent culture that responds to physiologic concentrations of estradiol. In contrast to the stimulatory effects of estrogen on gingival fibroblast proliferation, both collagen and noncollagen protein production were reduced when physiological concentrations of estradiol were introduced to fibroblasts in the culture. Female sex hormones also increase the rate of folate metabolism in the oral mucosa. Since folate is required for tissue maintenance, increased metabolism can deplete folate stores and inhibit tissue repair. Estrogen receptors beta $(E R \beta)$ on periodontal cells affect their immunoreactivity. They alter the antigen expression and presentation, cytokine production, as well as the expression of apoptotic factors and cell death. Studies have shown that progesterone stimulates the production of inflammatory mediator, prostaglandin E2, and enhances the accumulation of polymorphonuclear leukocytes in the gingival sulcus. Progesterone has also been found to enhance the chemotaxis of polymorphonuclear leukocytes, while low concentrations of estradiol have been demonstrated to reduce polymorphonuclear leukocyte chemotaxis. In addition, sex steroid hormones seem to modulate the production of cytokines, and progesterone has been shown to downregulate II- 6 production by human gingival fibroblasts to $50 \%$ of that of control values. ${ }^{7,8}$

\section{Oral Health Alterations during Menopause Burning Mouth Syndrome}

It is also referred to as glossodynia, glossopyrosis, glossalgia, stomatodynia, and stomatopyrosis. It is described as a condition characterized by intense pain and a spontaneous burning sensation in any areas of mouth, tongue, lips, and gums in the absence of any clinical and laboratory findings of the affected sites. This can be accompanied byother symptoms, like difficulty in swallowing, dysgeusia (alterations in taste sensation), xerostomia, and facial pain. Studies have found 50 to 60 years as the mean age of onset of this condition with more predilection in females(ranging from 3:1 to $16: 1$ ). This predilection increases with age, supporting the role of menopause. Studies have reported that its typical onset is 3 years prior to the beginning of menopause to 12 years after the onset of menopause. This condition can be caused by Candida albicans, staphylococci, streptococci, and anaerobes. It is also found in association with xerostomia, anxiety, depression, diabetes, and nutritional deficiency (iron and vitamin B complex). The local causes include oral carcinoma, ill-fitting dentures, and hypersensitivity to mercury and acrylic resins. ${ }^{9}$

Rabiei and associates conducted a study on 156 postmenopausal women. They concluded that postmenopausal women with psychological disorders suffer from burning mouth syndrome (BMS) and candidiasis, job status, denture, and xerostomia are associated factors in its etiology. Majority of other studies have similar results with a prevalence of $17.9-93 \%$ BMS in postmenopausal women. ${ }^{10}$ Emeline das Neves de Araújo Lima et al. conducted a study on 64 subjects with BMS and concluded that depression as well as reduced dehydroepiandrosteronelevels are associated factors for the development of BMS. ${ }^{11}$

BMS has a multifactorial etiology with no universally acceptable protocol. Therefore, its management should be comprehensive including hormone replacement therapy (HRT) and systemic and topical medication along with stress management. Various drugs like antidepressants, anxiolytics, anticonvulsants, antioxidants, local anesthetics, nonsteroidal antiinflammatory drugs, HRT, antimicrobials, mucosal protectants, salivary stimulants (sialogogues), and herbal supplements have been used systemically. Alternative treatment methods like lasers, acupuncture, behavioral therapies, yoga, relaxation therapy, meditation, group psychotherapy, and electroconvulsive therapy have also been tried. ${ }^{12}$ Lopez et al. conducted a randomized, double-blind, placebo-controlled trial which showed that the use of gabapentin alone (300 mg daily) or in combination with alpha lipoic acid (600 mg daily) was beneficial in reducing symptoms in 50 and $70 \%$ of patients with BMS, respectively, compared to placebo (15\%). Capsaicin used in the form of $0.25 \%$ capsules three times a day reduced severe pain in $93 \%$ of patients within 1 month. But, $32 \%$ of patients reported gastric pain as a side effect. ${ }^{13} \mathrm{~A}$ combination 
of drugs, such as clonazepam, gabapentin, and baclofen, was suggested by Brailo V et al. 2016. ${ }^{14}$

Capsaicin used topically in the form of oral rinse reduced the burning sensation in $75 \%$ of patients within 2 months without any side effects. Another randomized controlled study reported that topical use of clonazepam lozenges reduced pain in $66 \%$ of patients after 2 weeks and in $29 \%$ after 6 months. Topical application of $0.5 \mathrm{~mL}$ of aloe vera gel reduced the glossodynia in $70 \%$ of patients in a study conducted by Lopez-Jornet et al. in 2013. Bergdahl et al. in 1995 reported cognitive behavioral therapy as an effective treatment modality for BMS. In 2016, study conducted by Brailo et al. concluded that untreated BMS can cause emotional conditions, such as depression, anxiety, and pain-related catastrophizing. Catastrophizing is related to anxiety that is conceptualized as a negative cognitive-affective response to pain, which is a tendency to enlarge or exaggerate the value of the threat of pain sensation. This can be coupled with an inability to distract from the pain. Reassurance was found to be effective on pain perception in BMS patients by Matsuoko et al. in 2010 HRT has been beneficial in reducing symptoms associated with BMS in patients having estrogen receptors at an oral epithelial level. ${ }^{15-17}$

As there is no definitive cause for BMS, its treatment should be based on the cause. Multiple factors being responsible for causing BMS; the dental professional should provide symptomatic treatment along with proper explanation;nutritional deficiencies should be managed with replacement therapy;faulty dentures should be repaired;allergic materials should be avoided; and in cases of fungal diseases effective antifungal treatment should be provided. In cases where drugs taken by women are the cause, their physicians should be consulted for alternative druguse. ${ }^{18}$

\section{Gingival and Periodontal Disorders}

\section{Menopause and Gingival Health}

Estrogen increases epithelial keratinization and stimulates fibroblast proliferation and proliferation of basal epithelial cells. Hence, it increases the number of gingival epithelial cells. Accordingly, in postmenopausal women, there is a decrease in epithelial keratinization of the marginal gingiva and desquamation of the gingival tissues along with a decrease in the size of the gingiva. The gingiva appears to have a smooth surface with mottling. Decreased level of estrogen may exaggerate the response to dental plaques characterized by abnormal, painless, dry, shiny, thin gingival bleeding called concurrent senile atrophic gingivitis, which might progress to menopausal gingival stomatitis. ${ }^{19}$

\section{Menopause and Periodontal Health}

There are three types of estrogen hormones. Estrone (E1) is the estrogen hormone secreted in postmenopausal women. Estradiol (E2) is the most common and potent estrogen secreted by ovaries in childbearing age. Estriol (E3) is the main estrogen secreted during pregnancy. Receptors for steroid sex hormones are found in the periodontal tissues. Hence, these are significantly affected by the fluctuations, retention, and metabolic conversion of sex steroid hormones. A decrease in the level of estrogen during menopause leads to chronic inflammation of the gingiva and periodontium and alveolar bone loss. Osteoporosis of alveolar bone can lead to loss of tooth support in postmenopausal women. The resorption of bone following tooth extraction is greater after menopause. This causes problems during implant placement and denture construction. ${ }^{20}$

\section{Menopause and Osteoporosis}

Estrogen receptors are present in the osteoclast progenitorcells and multinucleated osteoclasts. Estrogen deficiency during menopause affects bone remodeling by increasing the osteoclastic resorption activity without a corresponding increase in the osteoblastic activity. As a result,bone resorption is greater than bone deposition, leading to loss of bone. In the earlier phase of menopause, there is a negative calcium balance with average loss of $-200 \mathrm{mg}$ of calcium daily in the first 3 to 4 years to $-45 \mathrm{mg}$ daily in the next 5 to 10 years. Secondary hyperparathyroidism accounts for malabsorption of calcium in postmenopausal women. The net result is osteoporosis which is characterized by decreased bone strength (bone strength = bone mineral density + bone quality). ${ }^{21}$

In women, two phases of bone loss are present. The first phase is due to estrogen deficiency. It occurs mainly in the trabecular bone. The second phase is seen 4 years later and is characterized by loss of both trabecular and cortical bones. The second type of bone loss is also seen in men and is age related. The alveolar bone of the jaws is trabecular bone. Osteoporotic changes in the oral cavity can be noticed as resorption of alveolar bone, loss of periodontal attachment to the tooth leading to increased tooth mobility, and finally loss of tooth. Bone marrow density of the alveolar crest andthe subcrestal alveolar bone is decreased. Studies conducted in postmenopausal women have shown that the residual ridge resorption following tooth extraction is more than in premenopausal women. Resorption of ridges leads to ill-fitting dentures or loose dentures, leading to ulcers in the mouth and problems withchewing and speech. ${ }^{22}$ Sultan and Rao in 2011 conducted a cross-sectional study in postmenopausal women to find arelationship between periodontal disease and bone mineral density. They concluded that age of the women, duration of menopause, and body mass index had a significant correlation with the bone marrow density, whereas the loss of alveolar bone and periodontal attachment had an insignificant correlation. Hence,menopause is just a risk indicator for diseases of periodontal tissues. ${ }^{23}$ Taguchi et al. ${ }^{9}$ conducted a study on 330 Japanese postmenopausal women with a mean age of 56.8 years. They studied the effect of the use of estrogen on tooth retention, oral bone height, and oral bone porosity. Their results showed that estrogen may promote tooth retention by strengthening the periodontal attachment surrounding the teeth, but not increasing oral bone height and not decreasing oral bone porosity. ${ }^{24}$ Deepa and Jain $^{22}$ conducted a cross-sectional survey to assess the periodontal health status in ninety postmenopausal women, aged 45 to 80 years, of Meerut city. They found that postmenopausal women are at risk of developing destructive periodontal disease if they do not maintain proper oral hygiene practices. ${ }^{25}$ A recent study conducted by Arias-Herrera and associates ${ }^{19}$ on 30 postmenopausal women assessed the periodontal clinical parameters, microbiological samples, and immunological variables. They found statistically significant differences between groups for clinical attachment level, probing pocket depth (PPD), IL-1 $\beta$, and IL-6. Smoking habits, deeper PPD, and higher II- 6 levels in nonmenopausal, hormone therapy user group tend to increase the IL-1ß gingival crevicular fluid levels. These findings were supported by serum estrogen levels. The variable levels were higher in the menopausal hormone therapy (MHT) user group. ${ }^{26}$

Periodontal diseases in postmenopausal women can be managed by maintaining proper oral hygiene practices. Mechanical removal of dental plaques with the help of toothbrush and 
toothpaste, interdental cleaning with the help of dental floss, regular dental examination, balanced diet, and avoiding smoking can help in preventing gingival and periodontal diseases in postmenopausal women. Vitamin D (400 IU) and calcium supplements (1200 mg) can help in dealing with osteoporosis. Dietary intake of calcium (700 mg/day) should be encouraged.

\section{Menopause - Saliva and Xerostomia}

Saliva is a viscous, clear, watery fluid secreted by the salivary glands of the oral cavity. It is composed of water, electrolytes, mucus, white blood cells, epithelial cells, enzymes, antimicrobial agents, such as secretory IgA, and lysozymes. It plays a significant role in oral health maintenance, lubrication, taste perception, mastication, bolus formation, enzymatic digestion, and swallowing. Daily around $750-1000 \mathrm{~mL}$ of saliva is produced by the glands. Xerostomia is defined as dry mouth. It can be due to the diminution of salivary flow. ${ }^{27}$ The salivary flow is decreased in postmenopausal women because of decreased production of estrogen. Reduced salivary flow affects the quality of life of women by interfering with basic daily functions, such as mastication, swallowing, and speech. Reduced antibacterial properties of saliva may accelerate infection by $C$. albicans, as well as cavities, tooth decay and periodontal diseases, halitosis, and burning mouth. ${ }^{28}$

Agha-Hosseini and associates ${ }^{29}$ studied the relationship of stimulated saliva $17 \beta$-estradiol and oral dryness (OD) feeling in menopause. A case-control study was carried out in 76 selected menopausal women, aged 41 to 77 years, with or without OD feeling. They concluded that there is a negative correlation between OD feeling severity and stimulated whole saliva $17 \beta$-estradiol in menopausal women. ${ }^{29}$ Agha-Hosseini and associates ${ }^{29}$ conducted a case-control study on 76 postmenopausal women. They studied the serum and stimulated whole saliva PTH in menopausal women with xerostomia. They found that the severity of OD was in positive correlation with the serum and stimulated whole saliva PTH, and with the saliva calcium levels in this group of menopausal women. ${ }^{30}$

Mirzaii and associates ${ }^{31}$ studied the stimulated and unstimulated saliva progesterone in 70 menopausal women, aged 42 to 78 years, with xerostomia. Their results showed that subjects with dry mouth had a decreased unstimulated salivary flow and salivary progesterone compared with those without dry mouth. They concluded that salivary progesterone level appears associated with xerostomia in menopause. ${ }^{31}$ Rukmini and associates ${ }^{32}$ studied the effect of menopause on saliva and dental health. They conducted a crosssectional study on 40 healthy postmenopausal women aged between 41 and 60 years. They found a noticeable diminution in salivary $\mathrm{pH}$ and flow rate in postmenopausal women, which resulted in increased gingival and periodontal problems along with dental caries. ${ }^{32}$ Cydejko et al. ${ }^{33}$ conducted a study on menopausal and premenopausal women to estimate the differences in selected physicochemical properties of saliva. The salivary flow rate in menopausal women was significantly lower, whereas the concentration of lysozymes and ionized calcium in the saliva of menopausal women was distinctly higher. They concluded that the differences in saliva properties observed in menopause can potentially affect the oral environment of women in this particular period, possibly increasing the risk of some pathological changes in the oral cavity. ${ }^{33}$

\section{Management of Oral Problems} Oral Bone-sparing Agents

The most commonly used oral bone-sparing agentsto prevent osteoporotic fractures arebisphosphonates, such as alendronate.
They act by inhibiting the activity of osteoclasts. Theyact directly on the cell as well as on the bone matrix. They increase the osteoblastic differentiation and inhibit osteoclast recruitment and activity. Hence, they can be used in the management of periodontal diseases. Their use during the management of periodontal diseases has shown to improve the periodontal status and bone formation. Other bone-sparingagents used for the management of osteoporosis are risedronate and ibandronate, intravenous bisphosphonates, selective estrogen receptor modulators, and strontium ranelate. Recently denosumab, a RANKL inhibitor, approved in 2010, is used in patients with osteoporosis. ${ }^{34}$ Risedronate therapy in women has shown significantly less plaque accumulation, less gingival inflammation, lower probing depths, less periodontal attachment loss, and greater alveolar bone levels. Correct dosage, delivery system, and target area of action should be the factors to be considered while selecting bone-sparing agents. ${ }^{35}$

\section{Menopausal Hormone Therapy}

It is also known as HRT. It is given in cases with deficiency in sex hormones. Estrogen replacement therapy (ET, ERT) is given in estrogen-deficient cases. Estrogen-progestogen therapy is advised in cases where a combination of estrogen and progesterone preparation is administered. ERT reduced the frequency of vasomotor symptoms(sleeping, psychic and concentration disorders, and hot flashes) by $75 \%$. The intensity of the symptoms is reduced by $87 \%$. ERT improves the quality of life and the health of postmenopausal women. The inflammation of the skin, gingival, and periodontal tissues is reduced. It also promotes epithelial regeneration, thereby improving the quality of skin and periodontium. According to the critical window hypothesis and healthy cell bias hypothesis, MHT improves cognitive functions. It reduces depression associated with menopause. ${ }^{36-39}$

MHT has shown a significant effect in reducing bone loss and osteoporosis by inhibiting the osteoclastic activity and loss of bone mass. Various studies have shown that MHT has a positive effect on improving the density of alveolar bone but they have not found any consistent effect on the attachment level and the pocket depth of the periodontal tissues. ET should be considered in the management of osteoporosis in women who are not able to tolerate bone-sparing agents, like bisphosphonates, raloxifene, and teriparatide. MHT is also associated with various serious side effects, such as an increased risk of endometrial cancer,breast cancer,ovarian cancer,venous thromboembolism, and cardiovascular diseases (e.g., stroke orcardiac arrest). Various studies have found that ET increases the risk for gallbladder diseases. It is found that estrogen increases the cholesterol saturation of bile, alters bile acid composition, and decreases bile flow, leading to gallstone formation. Considering the pros and cons of $\mathrm{MHT}$, it is recommended to use the lowest effective dose for the shortest amount of time. Absolute contraindications for systemic MHT include hormone-related cancer, active liver disease, history of hormone-induced venous thromboembolism, history of pulmonary embolism not caused by trauma, vaginal bleeding of unknown etiology, and pregnancy. Relative contraindications include chronic liver disease, severe hypertriglyceridemia, endometriosis, history of endometrial cancer, history of breast cancer, and coronary artery disease. ${ }^{40-45}$

\section{Oral Health Care}

Dental plaque is the major etiology for dental, gingival, and periodontal diseases. Chemical and mechanical control of dental plaque is the most important preventive treatment for the management of dental and oral health problems. Regular oral 
hygiene practices including correct method of toothbrushing using proper toothpaste can mechanically remove the plaque biofilm and prevent its accumulation. Interdental cleaning aids like dental floss, interdental brushes, and oral irrigators can help in maintaining good oral hygiene. Chemical plaque control can be used as an adjunctive to mechanical plaque control for effective plaque removal. Chlorhexidine mouthwash (0.1-0.5\%) is the most effective agent in chemical plaque control. However, it can cause irritation to oral tissues along with dryness if used inappropriately. ${ }^{46}$ Use of fluoride-containing toothpaste and mouthwash is recommended to prevent dental caries. Topical application of fluoride can provide protection from dentinal sensitivity. Saliva substitutes, artificial saliva, and salivary flow stimulators can be used in the management of xerostomia. Topical application of analgesic and anesthetic gels can be recommended for the management of BMS. Women should be encouraged to have a balanced diet, exercise, sleep, and meditation to overcome nutritional and psychological problems.

\section{Conclusion}

Oral health is the mirror of general health. Women are more sensitive to oral health disorders due to their hormonal changes. Menopause affects general health as well as oral health. Dentists, gynecologists, and physicians should work together for the management of oral, physical, and psychological problems associated with menopause. Postmenopausal women should be advised regular dental checkups along with good oral hygiene measures to maintain oral health.

\section{References}

1. Unni J. Third consensus meeting of Indian Menopause Society (2008): A summary. J Midlife Health 2010;1(1):43-47. DOI: 10.4103/09767800.66987.

2. Bansal R, Aggarwal N. Menopausal hot flashes: a concise review. J Midlife Health 2019;10(1):6-13. DOI: 10.4103/jmh.JMH_7_19.

3. Meeta, Digumarti L, Agarwal N, et al. Clinical practice guidelines on menopause: an executive summary and recommendations. J Midlife Health 2013;4(2):77-106. DOI: 10.4103/0976-7800.115290.

4. Sharma S. An update of menopausal hormone therapy: present perspective. Obstet Gynecol Int J 2018;9(2):124-125. DOI: 10.15406/ ogij.2018.09.00316.

5. Perlman B, Kulak D, Goldsmith LT, et al. The etiology of menopause: not just ovarian dysfunction but also a role for the central nervous system. Global Reprod Health 2018;3(2):e8. DOI: 10.1097/ GRH.0000000000000008.

6. Scardina GA, Messina P. Oral microcirculation in postmenopause: a possible correlation with periodontitis. Gerodontology 2012;29: e1045-e1051. PMID: 22212114. DOI: 10.1111/j.1741-2358.2011.00608.x.

7. Palacios S, Stevenson JC, Schaudig K, etal. Hormonetherapy for first-line management of menopausal symptoms: practical recommendations. Womens Health (Lond) 2019;15:1745506519864009. DOI: 10.1177/ 1745506519864009. PMID: 31378196. PMCID: PMC6683316.

8. Gambacciani M, Cagnacci A, Lello S. Hormone replacement therapy and prevention of chronic conditions. Climacteric 2019;22(3):303-306. DOI: 10.1080/13697137.2018.1551347.

9. Taguchi A, Sanada M, Suei Y, et al. Effect of estrogen use on tooth retention, oral bone height, and oral bone porosity in Japanese postmenopausal women. Menopause 2004;11(5):556-562. DOI: 10.1097/01.gme.0000113845.74462.bf.

10. Vaidya R. Burning mouth syndrome at menopause: elusive etiology. J Midlife Health 2012;3(1):3-4. DOI: 10.4103/0976-7800.98809. PMID: 22923972. PMCID: PMC3425145.

11. Araújo Lima EN, Barbosa NG, Santos ACS, et al. Comparative analysis of psychological, hormonal, and genetic factors between burning mouth syndrome and secondary oral burning. Pain Med 2016;17(9):1602-1611. PMID: 26849950. DOI: 10.1093/pm/pnv087.

12. Rabiei M, Leili EK, Alizadeh L. Burning mouth syndrome: a comparative cross-sectional study. Contemp Clin Dent 2018;9(Suppl S2):S256-S260. DOI: 10.4103/ccd.ccd_175_18.

13. Buencamino MC, Palomo L, Thacker HL. How menopause affects oral health, and what we can do about it. Cleve Clin J Med 2009;76(8):467475. DOI: 10.3949/ccjm.76a.08095.

14. Brailo V, Firić $M$, Vučićević Boras $\mathrm{V}$, et al. Impact of reassurance on pain perception in patients with primary burning mouth syndrome. Oral Dis 2016;22(6):512-516. DOI: 10.1111/odi.12493.

15. Lopez-Jornet P, Camacho-Alonso F, Molino-Pagan D. Prospective, randomized, double-blind, clinical evaluation of Aloe vera Barbadensis, applied in combination with a tongue protector to treat burning mouth syndrome. J Oral Pathol Med 2013;42(4):295-301. DOI: 10.1111/jop.12002.

16. Dahiya $P$, Kamal $R$, Kumar $M$, et al. Burning mouth syndrome and menopause. Int J Prev Med 2013;4(1):15-20. PMID: 23411996. PMCID: PMC3570906.

17. Mutneja P, Dhawan $P$, Raina A, et al. Menopause and the oral cavity. Indian J Endocrinol Metab 2012;16(4):548-551. DOI: 10.4103/22308210.98007.

18. Newadkar UR. Mouth on fire: oral discomfort in postmenopausal women may be surprising!! J Midlife Health 2015;6(4):184-186. DOI: 10.4103/0976-7800.172351. PMID: 26903760. PMCID: PMC4743282.

19. Arias-Herrera S, Bascones-llundian C, Bascones-Martínez A. Difference in the expression of inflammatory mediators in gingival crevicular fluid in postmenopausal patients with chronic periodontitis with and without menopausal hormone therapy. Eur J ObstetGynecol Reprod Biol X 2019;3:100021. Published 2019 Apr 18. DOI: 10.1016/j. eurox.2019.100021.

20. Bhardwaj A, Bhardwaj SV. Effect of menopause on women's periodontium. J Midlife Health 2012;3(1):5-9. DOI: 10.4103/09767800.98810.

21. Mascitelli L, Pezzetta F, Goldstein MR. Menopause, vitamin-D and oral health. Cleve Clin J Med 2009;76(11):629-630. DOI: 10.3949/ ccjm.76c.11001.

22. Deepa D, Jain G. Assessment of periodontal health status in postmenopausal women visiting dental hospital from in and around Meerut city: Cross-sectional observational study. J Midlife Health 2016;7(4):175-179. DOI: 10.4103/0976-7800.195696.

23. Sultan N, Rao J. Association between periodontal disease and bone mineral density in postmenopausal women: A cross sectional study. Med Oral Patol Oral Cir Bucal 2011;16(3):e440-e447. DOI:10.4317/ medoral.16.e440.

24. Mascarenhas P, Gapski R, Al-Shammari K, et al. Influence of sex hormones on the periodontium. J Clin Periodontol 2003;30(8):671681. DOI: 10.1034/j.1600-051x.2003.00055.x.

25. Tarkkila L, Kari K, Furuholm J, et al. Periodontal disease-associated micro-organisms in peri-menopausal and post-menopausal women using or not using hormone replacement therapy. A two-year follow-up study. BMC Oral Health 2010;10:10. DOI: 10.1186/1472-683110-10.

26. Tomar N, Gupta C, Kaushik M, et al. Nutrigenomics: a perio-nutrition interrelationship. J Oral Res Rev 2017;9(1):32-36. DOI: 10.4103/22494987.201401.

27. Güncü GN, Tözüm TF, Cağlayan F. Effects of endogenous sex hormones on the periodontium: review of literature. Aust Dent J 2005;50(3):138-145. DOI: 10.1111/j.1834-7819.2005.tb00352.x.

28. Prasanna JS, Sumadhura C, Karunakar P. A comparative analysis of preand postmenopausal females with periodontitis and its response to a non invasive clinical approach. J Menopausal Med 2017;23(3):202-209. DOI: 10.6118/jmm.2017.23.3.202. Epub 2017 Dec 29. PMID: 29354621. PMCID: PMC5770531.

29. Agha-Hosseini F, Mirzaii-Dizgah I, Mansourian A, et al. Relationship of stimulated saliva 17beta-oestradiol and oral dryness feeling in menopause. Maturitas 2009;62(2):197-199. DOI: 10.1016/j. maturitas.2008.10.016. 
30. Agha-Hosseini F, Mirzaii-Dizgah I, Mansourian A, et al. Serum and stimulated whole saliva parathyroid hormone in menopausal women with oral dry feeling. Oral Surg Oral Med Oral Pathol Oral Radiol Endod 2009;107(6):806-810. DOI: 10.1016/j.tripleo.2009.01.024.

31. Mirzaii-Dizgah I, Agha-Hosseini F. Stimulated and unstimulated saliva progesterone in menopausal women with oral dryness feeling. Clin Oral Invest 2011;15(6):859-862. DOI: 10.1007/s00784-010-0449-z.

32. Rukmini JN, Sachan R, Sibi N, et al. Effect of menopause on saliva and dental health. J Int Soc Prevent Communit Dent 2018;8(6):529-533. DOI: 10.4103/jispcd.JISPCD_68_18.

33. Cydejko A, Kusiak A, Grzybowska ME, et al. Selected physicochemical properties of saliva in menopausal women-a pilot study. Int J Environ Res Public Health 2020;17(7):2604. DOI: 10.3390/ ijerph17072604.

34. Ferguson S, FeudjoTepie M, Taylor A, et al. The impact of persistence with bisphosphonates on health resource utilization and fracture risk in the UK: a study of patient records from the UK Clinical Practice Research Datalink. J Eval Clin Pract 2016;22(1):31-39. DOI: 10.1111/jep.12422.

35. Fait T.Menopause hormone therapy: latest developments and clinical practice. Drugs Context 2019;8:212551. DOI: 10.7573/dic.212551.

36. Pizzo G, Guiglia R, Licata ME, et al. Effect of hormone replacement therapy (HRT) on periodontal status of postmenopausal women. Med Sci Monit 2011;17(4):PH23-PH27. DOI: 10.12659/MSM.881700.

37. Alves RC, Félix SA, Rodriguez-Archilla A, et al. Relationship between menopause and periodontal disease: a cross-sectional study in a Portuguese population. Int J Clin Exp Med 2015;8(7):11412-11419. PMID: 26379957. PMCID: PMC4565340.

38. Warren MP, Shu AR, Dominguez JE. Menopause and hormone replacement. [Updated 2015 Feb 25]. In: Feingold KR, Anawalt B,
Boyce A, et al., editors. Endotext [Internet]. South Dartmouth (MA): MDText.com, Inc.; 2000. Available from: https://www.ncbi.nlm.nih. gov/books/NBK279050/.

39. Steffens JP, Valenga HM, Santana LCL, et al. Role of testosterone and androgen receptor in periodontal disease progression in female rats. J Periodontol 2019;91(4):545-553. DOI: 10.1002/JPER.19-0099.

40. Nayak G, Kamath A, Kumar P, et al. A study of quality of life among perimenopausal women in selected coastal areas of Karnataka, India. J Midlife Health 2012;3(2):71-75. DOI: 10.4103/0976-7800.104456.

41. Shigli KA, Giri PA. Oral manifestations of menopause. J Basic Clin Reprod Sci 2015;4(1):4-8. DOI: 10.4103/2278-960X.153514.

42. Parakh D, Garia DK, Dagli R, et al. Evaluation of the effect of menopause on saliva and dental health. Int J Oral Health Dent 2016;2(2):71-76. DOI: 10.5958/2395-499X.2016.00012.5.

43. Siregar MFG. Menopause and the oral cavity: an oral hygiene update in Indonesia. Int J Community Med Public Health 2015;2(3):210-216. DOI: 10.18203/2394-6040.ijcmph20150474.

44. Burkard T, Moser M, Rauch M, et al. Utilization pattern of hormone therapy in UK general practice between 1996 and 2015: a descriptive study. Menopause 2019;26(7):741-749. DOI: 10.1097/ GME.0000000000001300.

45. Nudy, M, Chinchilli, VM, Foy, AJ. A systematic review and metaregression analysis to examine the 'timing hypothesis' of hormone replacement therapy on mortality, coronary heart disease, and stroke. Int J Cardiol Heart Vasc 2019;22:123-131. DOI: 10.1016/j. ijcha.2019.01.001.

46. Mlachkova AM, Popova CL. Efficiency of nonsurgical periodontal therapy in moderate chronic periodontitis. Folia Med (Plovdiv) 2014;56(2):109-115. DOI: 10.2478/folmed-2014-0016. 